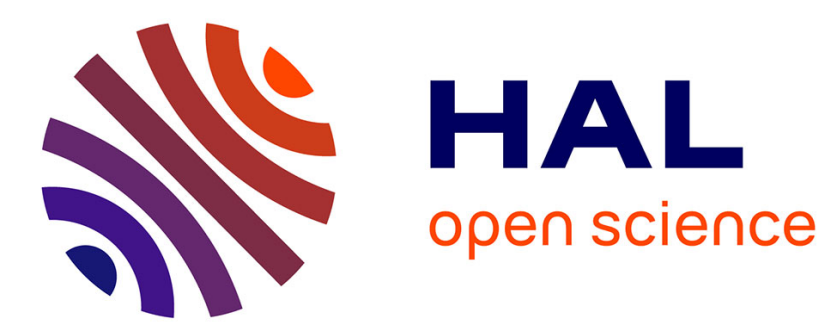

\title{
Optimization of an autodyne laser interferometer for high-speed confocal imaging
}

Eric Lacot, Wilfried Glastre, Olivier Jacquin, Olivier Hugon, Hugues Guillet de Chatellus

\section{- To cite this version:}

Eric Lacot, Wilfried Glastre, Olivier Jacquin, Olivier Hugon, Hugues Guillet de Chatellus. Optimization of an autodyne laser interferometer for high-speed confocal imaging. Journal of the Optical Society of America. A Optics, Image Science, and Vision, 2012, 30 (1), pp.60-70. hal-00768452

\section{HAL Id: hal-00768452 \\ https://hal.science/hal-00768452}

Submitted on 21 Dec 2012

HAL is a multi-disciplinary open access archive for the deposit and dissemination of scientific research documents, whether they are published or not. The documents may come from teaching and research institutions in France or abroad, or from public or private research centers.
L'archive ouverte pluridisciplinaire HAL, est destinée au dépôt et à la diffusion de documents scientifiques de niveau recherche, publiés ou non, émanant des établissements d'enseignement et de recherche français ou étrangers, des laboratoires publics ou privés. 


\title{
Optimization of an autodyne laser interferometer for high- speed confocal imaging
}

\author{
Eric Lacot," Wilfried Glastre, Olivier Jacquin, Olivier Hugon, \\ and Hugues Guillet de Chatellus \\ Centre National de la Recherche Scientifique/ Université de Grenoble 1, Laboratoire \\ Interdisciplinaire de Physique, UMR 5588, Grenoble F- 38402, France \\ *Corresponding author: eric.lacot@ujf-grenoble.fr
}

In autodyne interferometry, the beating between the reference beam and the signal beam takes place inside the laser cavity and therefore the laser fulfills simultaneously the roles of emitter and detector of photons. In these conditions, the laser relaxation oscillations play a leading role, both in the laser quantum noise which determines the signal to noise ratio (SNR) and also in the laser dynamics which determines the response time of the interferometer. In the present study, we have theoretically analyzed the SNR and the response time of a Laser Optical Feedback Imaging (LOFI) setup based on an autodyne interferometer. More precisely, we have compared the images quality of two lasers having the same output power, the same relaxation frequency, but having two different values of the LOFI gain induced by two different values of the laser response time. From this study, we have finally determined the best laser dynamical parameters and the best experimental conditions for high speed imaging at the shot noise limit. Finally, we conclude that a laser diode with a very short response time (in the nanosecond range) seems to be an interesting 
candidate compared to solid-state microchip laser with a response time of several tens of microsecond. Analytical predictions are confirmed by numerical simulations.

(C) 2012 Optical Society of America

OCIS codes: $110.3175,280.3420$. 


\section{INTRODUCTION}

When a frequency shift is introduced between the two beams of an interferometer, one realizes the so-called heterodyne interferometry. Resulting from this shift, the interference between the two waves produces an intensity modulation at the beat frequency, which can be measured by a photo-detector. In this paper, we refer only to autodyne laser interferometry where the heterodyne wave mixing takes place inside the cavity of the laser source and is finally indirectly detected by a photodiode.

Since the development of the first laser in 1960, laser heterodyne interferometry has become a useful technique on which many high accuracy measurement systems for scientific and industrial applications are based [1]. Since the pioneer work of K. Otsuka, on self-mixing modulation effects in class-B laser [2] the sensitivity of laser dynamics to frequency-shifted optical feedback has been used in autodyne interferometry and metrology [3], for example in self-mixing laser Doppler velocimetry [4-7], vibrometry [8-10], near field microscopy [11,12] and laser optical feedback imaging (LOFI) experiments [13-16]. Compared to conventional optical heterodyne detection, frequency-shifted optical feedback shows an intensity modulation contrast higher by several orders of magnitude and the maximum of the modulation is obtained when the shift frequency is resonant with the laser relaxation oscillation frequency [17]. In this condition, an optical feedback level as low as $-170 \mathrm{~dB}$ (i.e. $10^{17}$ times weaker than the intracavity power) has been detected [5].

In previous papers [17-19], we have demonstrated that in autodyne interferometry, the main advantage of the resonant gain (defined by the ratio between the cavity damping rate and the population-inversion damping rate of the laser) is to raise the laser quantum noise over the detector noise in a relatively large frequency range around to the laser relaxation frequency. 
Moreover if the detection bandwidth is narrower than the laser dynamical relaxation width, the signal to noise ratio (SNR) of a LOFI setup is frequency independent, and more importantly, shot noise limited. We have also established that to maximize the dynamical range of a LOFI setup, the best value of the shift frequency is not the relaxation frequency, but the frequency at which the amplified laser quantum noise is equal to the detection noise level $[18,19]$.

For high-speed imaging, we need to decrease the signal acquisition time of our LOFI setup and therefore to work with a detection bandwidth larger than the laser dynamical relaxation bandwidth. Under this condition the laser transient dynamic cannot be ignored and the LOFI SNR becomes frequency dependent. The main objective of this paper is to determine the best laser dynamical parameters and the best experimental conditions to obtain high quality images (i.e. shot-noise limited) as fast as possible.

This paper is organized as follows. Firstly, after a basic description of our LOFI set-up (i.e. our autodyne interferometer) for confocal imaging, we briefly recall the expression of the LOFI permanent signal induced by the beating inside the laser cavity. Secondly, we determine the stationary LOFI SNR for different values of the experimental acquisition time compare to the laser dynamical response time. Thirdly, for autodyne imaging, we calculate the level of the transient LOFI signal appearing when during the laser scanning (i.e. from one image pixel to the next one), the target under investigation presents discontinuous physical properties. Finally, we determine the best laser parameters for high speed autodyne imaging with a shot-noise limited detection (i.e. highest quality image as fast as possible). In each section, analytical predictions are confirmed by numerical simulations. 


\section{AUTODYNE SIGNAL}

\section{A. LOFI set up}

A schematic diagram of the LOFI experimental setup (i.e. the autodyne experimental interferometer) is shown in Fig. 1. Typically the laser is a CW microchip with an output power $P_{\text {out }}$ of several milliwatts and a typical relaxation oscillation frequency $F_{R}$ in the megahertz range and a damping rate of the relaxation oscillation $\left(1 / \tau_{R}\right)$ in the kilohertz range [19-20]. The laser is therefore a class-B laser $\left(F_{R}>1 / \tau_{R}\right)$. The laser beam is sent on the target, through a frequency shifter. A part of the light diffracted and/or scattered by the target is then reinjected inside the laser cavity after a second pass through the frequency shifter. Therefore, the optical frequency of the reinjected light is shifted by $F_{e}$. This frequency can be adjusted and is typically of the order of the laser relaxation frequency $F_{R}$. For the geometrical point of view, the laser beam waist and the laser focal spot on the target under investigation are optically conjugated. At this point, one can already notice that, compared to a conventional heterodyne setup, the autodyne setup shown here does not require complex alignment. Indeed, the LOFI setup is even always self-aligned because the laser simultaneously fulfills the function of the source (i.e. photons-emitter) and of the photo-detector (i.e. photons-receptor).

The optical feedback is characterized by the electric field complex reflectivity $\left(r_{e}=\sqrt{R_{e}} \exp \left(j \phi_{e}\right)\right)$ of the target, where the phase $\phi_{e}$ describes the optical round trip between the laser and the target, while the effective power reflectivity $\left(R_{e}=\left|r_{e}\right|^{2}\right)$ takes into account the target albedo, the numerical aperture of the collection optics, the frequency shifters efficiencies and the transmission of all optical components (except for the beam splitter which is addressed 
separately) and the overlap of the retro-diffused field with the Gaussian cavity beam (confocal feature).

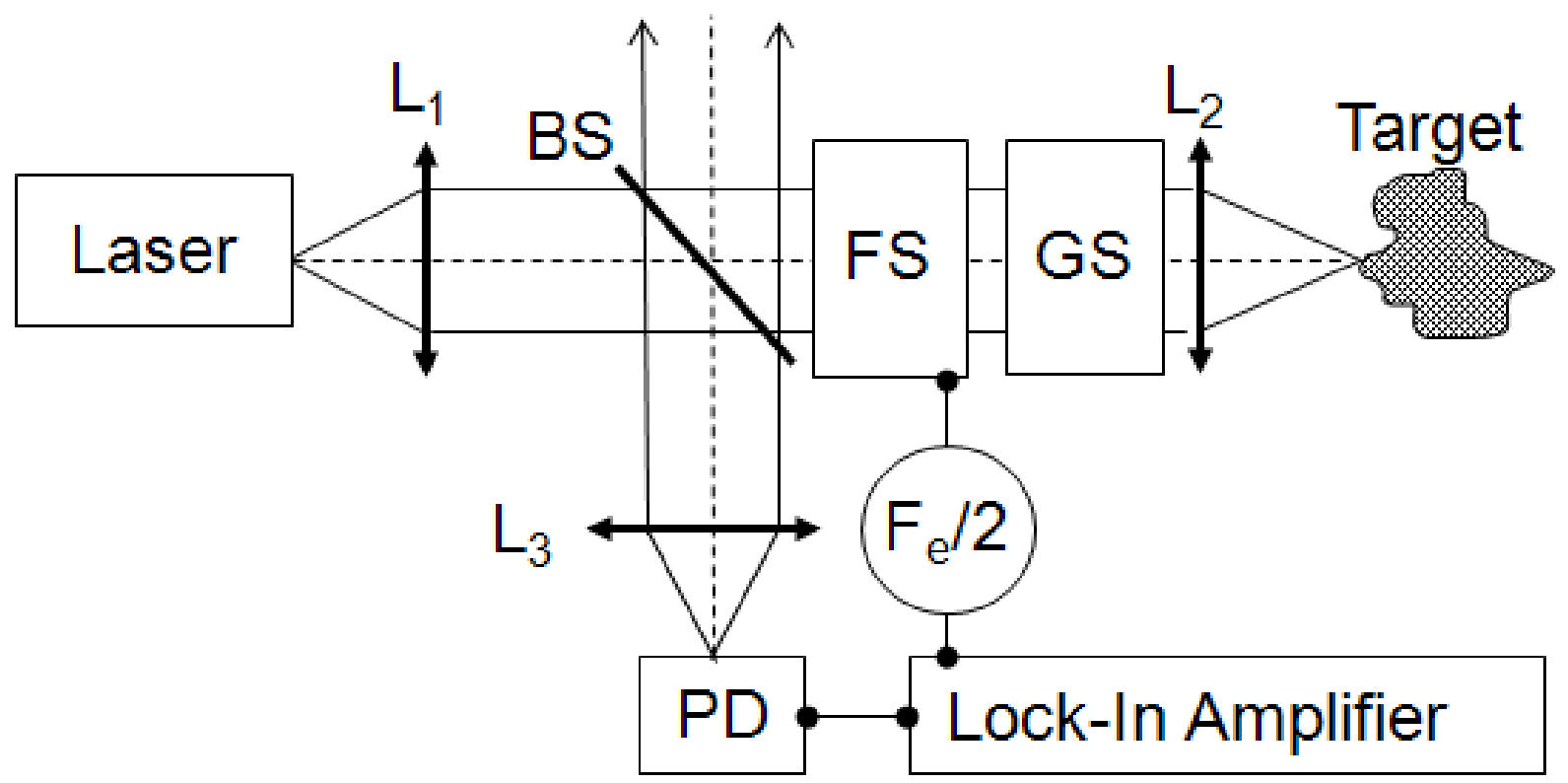

Fig. 1. Schematic diagram of the LOFI interferometer setup for scanning microscopy. $L_{1}, L_{2}$ and $L_{3}$ : Lenses, BS: Beam Splitter with a power reflectivity $R_{\text {bs }}$, GS: Galvanometric Scanner, FS Frequency Shifter with a round trip frequency-shift $F_{e}$, PD: Photodiode with a white noise spectrum. The lock-in amplifier is characterized by its integration time $T_{\text {int }}$. The laser is characterized by its output power $p_{\text {out }}$ (photons/s), its relaxation frequency $F_{R}$ and its dynamical response time $\tau_{R}$. The target is characterized by its effective reflectivity $R_{e}<<1$.

The coherent interaction (beating) between the lasing electric field and the frequency-shifted reinjected field leads to a modulation of the laser output power. For the detection purpose, a fraction of the output beam of the microchip laser is sent to a photodiode by means of a beam splitter characterized by a power reflectivity $\mathrm{R}_{\text {bs }}$. The photodiode is assumed to have a quantum efficiency of $100 \%$. The voltage delivered by the photodiode is finally analyzed by a lock-in amplifier which gives the LOFI signal (i.e. the magnitude and the phase of the retro-diffused electric field) at the demodulation frequency $F_{e}[15,16]$. The lock-in amplifier is characterized by its integration time $T_{\text {int }}$. Experimentally, the LOFI images are obtained pixel by pixel (i.e. 
point by point, line after line) by a full 2D galvanometric scanning and the necessary time needs to obtain an image composed of $N$ pixels is roughly given by: $N \times T_{\text {int }}$. For high speed imaging (i.e. high cadence imaging), one needs to use a value of $T_{\mathrm{int}}$ as small as possible. To determine the SNR of the obtained LOFI images, $T_{\text {int }}$ needs to be compared with the response time of the class-B laser $\left(\tau_{R}\right)$. In this paper, whatever the temporal values of $T_{\text {int }}$ (in the millisecond or microsecond range), we refer to a fast response time laser when: $\tau_{R}<<T_{\text {int }}$ and to a slow response time laser when: $\tau_{R} \gg T_{\text {int }}$.

\section{B. LOFI Modelling}

In the case of weak $\left(R_{e}<<1\right)$ frequency shifted optical feedback, the dynamical behavior of a reinjected solid-state laser can be described by the following set of equations $[10,17,18]$ :

$$
\begin{gathered}
\frac{d N}{d t}=\gamma_{1} N_{0}-\gamma_{1} N-B N\left|E_{c}\right|^{2}+F_{N}(t), \\
\frac{d E_{c}}{d t}=\frac{1}{2}\left(B N-\gamma_{c}\right) E_{c}+\gamma_{c} \sqrt{R_{e}}\left(1-R_{b s}\right) E_{c} \cos \left[2 \pi F_{e} t+\phi_{e}\right]+F_{E_{c}}(t),
\end{gathered}
$$

where, $\mathrm{N}$ is the population inversion, $E_{c}$ is the slowly varying amplitude of the laser electric field, B is related to the Einstein coefficient, $\gamma_{1}$ is the decay rate of the population inversion, $\gamma_{\mathrm{c}}$ is the laser cavity decay rate and $\gamma_{1} \mathrm{~N}_{0}$ is the pumping rate. Regarding the noise, the laser quantum fluctuations are described by the conventional Langevin noise functions $\mathrm{F}_{\mathrm{N}}(\mathrm{t})$ and $\mathrm{F}_{\mathrm{E}_{\mathrm{c}}}(\mathrm{t})$, which have a zero mean value and a white noise type correlation function [21-23]. 
The laser model presented above can be applied to three levels or four levels lasers with the condition that the lifetime of the upper level of the pumping transition is very short compared to the lifetime of the upper level of the laser transition. For example, this is condition is satisfied in a three levels laser such as erbium lasers as well as for a four levels laser such as neodymium laser. In the set of Eqs. (1), the feedback time delay $(\tau)$, linked to the optical round trip between the laser and the target is completely neglected. It means, that we only consider the case where the round trip time is shorter than the frequency shift $\left(2 \pi F_{e} \tau<<1\right)$.

\section{LOFI stationary signal}

In the set of Eqs. (1), the periodic functions express the beating (i.e. the coherent interaction) between the lasing and the feedback electric fields. The net gain of the laser is then modulated by the re-injected light at the optical shift frequency $\mathrm{F}_{\mathrm{e}}$. In the linear regime, the photon output rate $\mathrm{p}_{\text {out }}(\mathrm{t})=\gamma_{\mathrm{c}}\left|\mathrm{E}_{\mathrm{c}}(\mathrm{t})\right|^{2}$ (number of photons per second) is therefore periodically modulated [17]:

$$
p_{\text {out }}\left(t, F_{e}, R_{e}\right)=\left\langle p_{\text {out }}\right\rangle+2 G\left(F_{e}\right)\left(1-R_{b s}\right) \sqrt{R_{e}}\left\langle p_{\text {out }}\right\rangle \cos \left(2 \pi F_{e} t+\Phi_{e}\right)
$$

where $\left\langle p_{\text {out }}\right\rangle=\gamma_{c} \frac{\gamma_{1}}{B}(r-1)$ is the mean value the photon output rate with $r=\frac{N_{0}}{\gamma_{c} / B}$ the normalized pumping parameter. In Eq. (2), $G\left(F_{e}\right)$ describes the amplification gain of the autodyne waves mixing with:

$$
G\left(F_{e}\right)=\gamma_{c} \frac{\sqrt{\left(2 / \tau_{R}\right)^{2}+\left(2 \pi F_{e}\right)^{2}}}{\sqrt{\left[\left(2 \pi F_{R}\right)^{2}-\left(2 \pi F_{e}\right)^{2}\right]^{2}+\left(2 / \tau_{R}\right)^{2}\left(2 \pi F_{e}\right)^{2}}} \underset{F_{R} \gg \frac{1}{\tau_{R}}}{\approx} \frac{\gamma_{c}}{2} \frac{1}{\sqrt{\left(2 \pi F_{R}-2 \pi F_{e}\right)^{2}+\left(1 / \tau_{R}\right)^{2}}}
$$


where for a class-B laser $\left(F_{R}>>1 / \tau_{R}\right), F_{R}=\Omega_{R} / 2 \pi=\sqrt{\gamma_{1} \gamma_{c}(r-1)} / 2 \pi$ is the frequency of the laser relaxation oscillations and $\tau_{R}=2 /\left(\gamma_{1} r\right)$ is the damping time of the relaxation oscillations [23]. At this point one can notice that for a class-B laser, $\tau_{R}$ which is very long compared to the photon lifetime in the cavity $\left(\tau_{R}>1 / \gamma_{c}\right)$, is therefore the laser characteristic response time [23]. In a LOFI interferometer, a particularly interesting situation is the resonance case $\left(F_{e}=F_{R}\right)$ where the LOFI signal gain (i.e. the autodyne gain) becomes:

$$
G\left(F_{R}\right)=\frac{\gamma_{c} \tau_{R}}{2}
$$

For a microchip laser, this ratio is typically of the order of $10^{4}-10^{6}[17,19]$ and the main advantage of the LOFI detection technique seems to come from this resonant amplification of the optical wave mixing $[3,13,17]$.

Using Eq. (2), one can define the modulation contrast (MC) of the autodyne wave mixing:

$$
M C\left(F_{e}, R_{e}\right)=\frac{\Delta p_{\text {out }}\left(F_{e}, R_{e}\right)}{\left\langle p_{\text {out }}\right\rangle}=2 G\left(F_{e}\right)\left(1-R_{b s}\right) \sqrt{R_{e}} .
$$

In a LOFI experiment, because the laser simultaneously fulfills the functions of the source and of the detector, we assume to simply define the saturation level as the effective reflectivity corresponding to a maximum modulation of the laser output power $(\mathrm{MC}=1)$ :

$$
R_{\text {Sat }}\left(F_{e}\right)=\frac{1}{4} \times \frac{1}{G^{2}\left(F_{e}\right)} \times \frac{1}{\left(1-R_{b s}\right)^{2}}
$$

Finally, using a lock-in amplifier, the LOFI signal at the demodulation frequency $\mathrm{F}_{\mathrm{e}}$ is given by: 


$$
S_{\text {LOFI }}\left(F_{e}, R_{e}\right)=R_{b s} \frac{\Delta p_{\text {out }}\left(F_{e}, R_{e}\right)}{\sqrt{2}}=R_{b s} \frac{2 G\left(F_{e}\right)\left(1-R_{b s}\right) \sqrt{R_{e}}\left\langle p_{\text {out }}\right\rangle}{\sqrt{2}} .
$$

\section{STATIONARY SNR OF AN AUTODYNE INTERFEROMETER}

\section{A. Stationary LOFI SNR}

Without optical feedback $\left(R_{e}=0\right)$, the set of Eqs. (1) allows us to study the laser quantum fluctuations induced by the Langevin noise terms $\left(F_{N}(t)\right.$ and $\left.F_{E_{c}}(t)\right)$. Using the WienerKhintchine theorem, on can obtain the power density spectrum of the laser output power quantum fluctuations $[17,21,22]$ :

$$
P D_{\text {Laser }}(F)=2\left\langle p_{\text {out }}\right\rangle \gamma_{c}^{2} \frac{\left(2 / \tau_{R}\right)^{2}+(2 \pi F)^{2}}{\left[\left(2 \pi F_{R}\right)^{2}-(2 \pi F)^{2}\right]^{2}+\left(2 / \tau_{R}\right)^{2}(2 \pi F)^{2}}=2\left\langle p_{\text {out }}(t)\right\rangle G^{2}(F) .
$$

The LOFI noise power obtained after the photodiode detection (i.e. after the beam splitter reflection) and the lock-in amplifier filtering at the modulation frequency $\left(F_{e}\right)$ is then given by:

$$
N_{\text {Laser }}^{2}\left(F_{e}, T_{\text {int }}\right)=2 R_{b s} \int_{-\infty}^{+\infty} P D_{\text {Laser }}(F)\left|F_{\text {int }}\left(F-F_{e}, T_{\text {int }}\right)\right|^{2} d F
$$

where for an integration time $T_{\text {int }}$ :

$$
\left|F_{\text {int }}\left(F, T_{\text {int }}\right)\right|^{2}=\frac{1}{T_{\text {int }}^{2}} \frac{1}{\frac{1}{T_{\text {int }}^{2}}+(2 \pi F)^{2}}
$$


is assumed to be a first order power filter .

By combining Eqs. (8) and (9), one finally obtains for a class-B laser $\left(F_{R}>>\frac{1}{\tau_{R}}\right)$, the following analytical expression of LOFI noise induced by laser quantum noise:

$$
N_{\text {Laser }}^{2}\left(F_{e}, T_{\mathrm{int}}\right)=R_{b s}\left\langle p_{\text {out }}(t)\right\rangle \frac{1}{2} \frac{\tau_{R}}{T_{\mathrm{int}}}\left[\frac{1}{T_{\mathrm{int}}}+\frac{1}{\tau_{R}}\right] \frac{\gamma_{c}^{2}}{\left[\left\lceil\frac{1}{T_{\mathrm{int}}}+\frac{1}{\tau_{R}}\right]^{2}+\left(2 \pi F_{e}-2 \pi F_{R}\right)^{2}\right]},
$$

and by combining Eqs. (7) and (10), one finally obtains the stationary LOFI SNR:

$$
\operatorname{SNR}\left(F_{e}, R_{e}, T_{\mathrm{int}}\right)=\frac{S_{\text {LOFI }}\left(F_{e}, R_{e}\right)}{N_{\text {Laser }}\left(F_{e}, T_{\mathrm{int}}\right)} .
$$

Fig. 2 shows the evolution of the stationary LOFI SNR $\left(S_{\text {LOFI }} / N_{\text {Laser }}\right)$ versus the normalized shift frequency $\left(F_{e} / F_{R}\right)$ for different values of the lock-in integration time $\left(T_{\text {int }}\right)$ compared to laser response time $\left(\tau_{R}\right)$. 


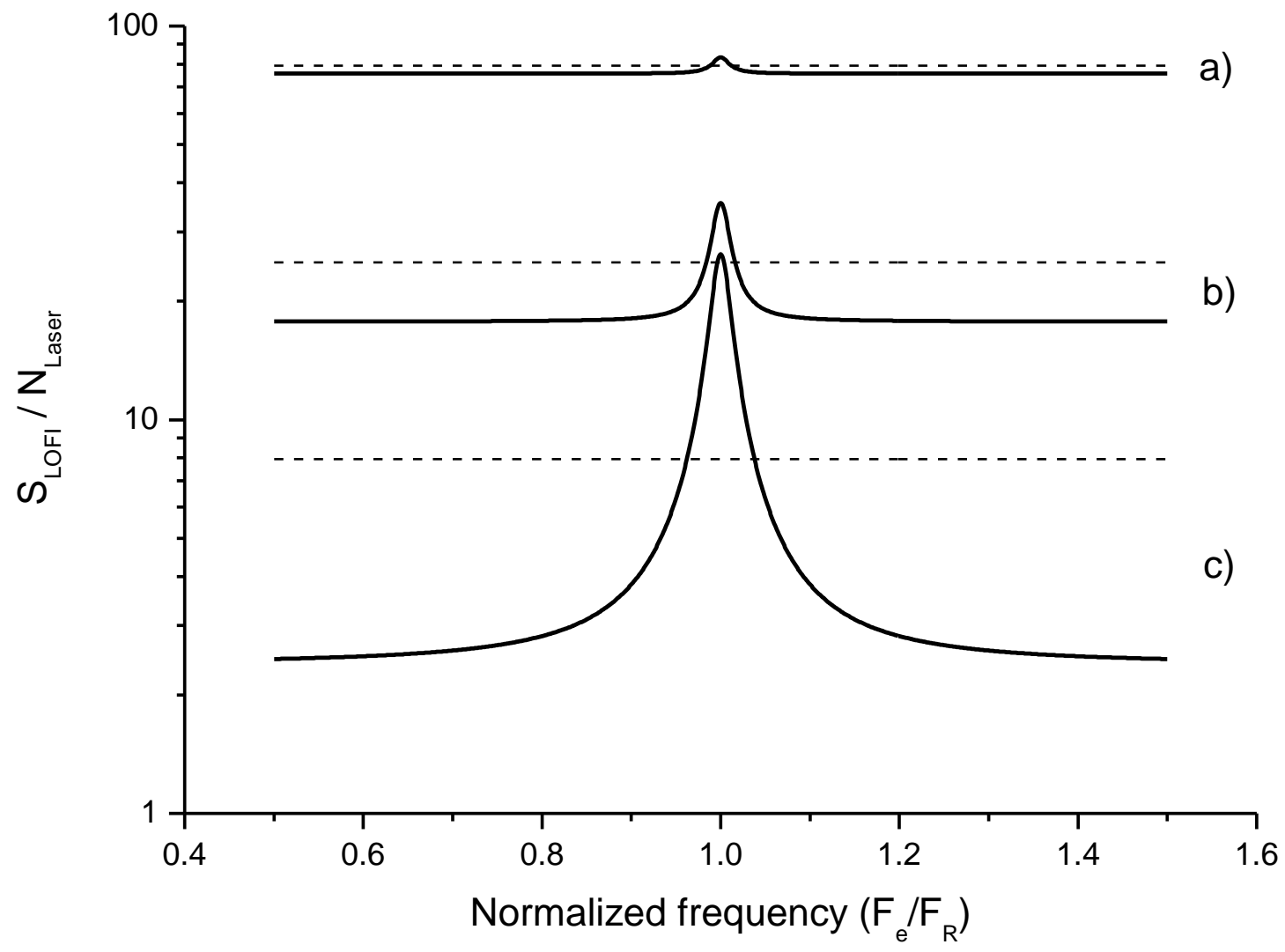

Fig. 2. Stationary LOFI SNR ( $S_{L O F I} / N_{\text {Laser }}$ ) versus the normalized shift-frequency $\left(F_{e} / F_{R}\right)$ for different values of the lock-in integration time: a) $T_{\text {int }}=10 \times \tau_{R}$, b) $T_{\text {int }}=\tau_{R}$ c) $T_{\text {int }}=\tau_{R} / 10$. The experimental conditions are $\mathrm{R}_{\mathrm{e}}=2 \times 10^{-11}$ and $\mathrm{R}_{\mathrm{bs}}=1 / 2$. The laser is a class-B laser with: $p_{\text {out }}=3.2 \times 10^{17}$ photons $/ \mathrm{s} \quad\left(P_{\text {out }}=60 \mathrm{~mW}\right.$ at $\left.\lambda=1064 \mathrm{~nm}\right) \quad F_{R}=356 \mathrm{kHz} \quad$ and $\quad F_{R} \tau_{R} \approx 14$ $\left(r=1.02, \gamma_{c}=5 \times 10^{9} s^{-1}, \gamma_{1}=5 \times 10^{3} s^{-1}\right)$. For each integration time the continuous line shows the exact value of the LOFI SNR [Eq. (11)], while the dash line shows the corresponding LOFI shot-noise limit [Eq. (13)].

\section{B. Acquisition with a fast response time laser $\left(T_{\mathrm{int}}>\tau_{R}\right)$}

If the lock-in integration time is long compare to the laser response time $\left(T_{\text {int }}>>\tau_{R}\right)$, the noise [Eq. (10)] is then simply given by:

$$
N_{\text {Laser }}\left(F_{e}, T_{\text {int }}>>\tau_{R}\right) \approx 2 \sqrt{R_{b s}\left\langle p_{\text {out }}(t)\right\rangle} \frac{\gamma_{c}}{2} \frac{1}{\sqrt{\left(2 \pi F_{R}-2 \pi F_{e}\right)^{2}+\left(1 / \tau_{R}\right)^{2}}} \frac{1}{\sqrt{2 T_{\mathrm{int}}}}
$$


or identically, by introducing the expression of the gain given by Eq. (3):

$$
N_{\text {Laser }}\left(F_{e}, T_{\mathrm{int}}>\tau_{R}\right) \approx 2 \sqrt{R_{b s}\left\langle p_{\text {out }}(t)\right\rangle} G\left(F_{e}\right) \frac{1}{\sqrt{2 T_{\mathrm{int}}}} .
$$

At this point, one can notice that the resonant amplification gain $G\left(F_{e}\right)$ present in the LOFI signal [see Eq. (7)], is also present in the LOFI noise and, as a result, the SNR of the LOFI setup is frequency independent:

$$
\operatorname{SNR}\left(F_{e}, R_{e}, T_{\mathrm{int}}>\tau_{R}\right)=\sqrt{R_{e}}\left(1-R_{b s}\right) \times \sqrt{R_{b s}\left\langle p_{\text {out }}\right\rangle T_{\mathrm{int}}},
$$

As mentioned above, the relaxation frequency seems to be of no particular importance [see Fig. 2(a) for comparison]. At this point, one can notice that the condition: $\operatorname{SNR}\left(F_{e}, R_{e, \text { min }}, T_{\mathrm{int}}>\tau_{R}\right)=1$, physically, means that during the integration time $\left(T_{\mathrm{int}}\right)$, only $1 / R_{\text {bs }}$ back-reflected photons are detected:

$$
R_{e, \text { min }}\left(1-R_{b s}\right)^{2}\left\langle p_{\text {out }}\right\rangle T_{\text {int }}=\frac{1}{R_{b s}} .
$$

The LOFI set-up is therefore shot noise limited and the beam splitter reflectivity $\left(\mathrm{R}_{\mathrm{bs}}\right)$ appearing in Eqs. (7) and (10) and finally in the right hand term of Eq. (14), can be interpreted as the quantum efficiency of the LOFI detection where the laser modulation produced inside the laser cavity signal is finally indirectly detected by the photodiode after the beam splitter. Consequently, and throughout the rest of the manuscript, Eq. (13) is what we will call the LOFI shot noise limit. 


\section{Acquisition with a slow response time laser $\left(T_{\text {int }}<<\tau_{R}\right)$}

If now the lock-in integration time is short compared to the laser response time $\left(T_{\mathrm{int}}<<\tau_{R}\right)$, the laser quantum noise [Eq. (10)] is then approximately given by:

$$
N_{\text {Laser }}\left(F_{e}, T_{\mathrm{int}} \ll<\tau_{R}\right) \approx 2 \sqrt{R_{b s}\left\langle p_{\text {out }}(t)\right\rangle} \frac{\gamma_{c}}{\sqrt{\left(\frac{2}{T_{\text {int }}}\right)^{2}+4\left(2 \pi F_{e}-2 \pi F_{R}\right)^{2}}} \frac{1}{\sqrt{2 T_{\mathrm{int}}}} \sqrt{\frac{\tau_{R}}{T_{\mathrm{int}}}}
$$

Now, to analyze the LOFI SNR, three different cases need to be studied depending on the interval between the shift frequency and the relaxation frequency.

Firstly, if we work very far away from resonance $\left(\left|\left(F_{e}-F_{R}\right)\right|>>\frac{1}{T_{\mathrm{int}}}>>\frac{1}{\tau_{R}}\right)$, then the LOFI noise [Eq. (15)] and LOFI signal [Eq. (7)] can respectively and approximately be given by:

$$
\begin{gathered}
N_{\text {Laser }}\left(F_{e}, T_{\text {int }}<<\tau_{R}\right) \approx 2 \sqrt{R_{b s}\left\langle p_{\text {out }}(t)\right\rangle} \frac{\gamma_{c}}{4\left|2 \pi F_{e}-2 \pi F_{R}\right|} \frac{1}{\sqrt{2 T_{\mathrm{int}}}} \sqrt{\frac{\tau_{R}}{T_{\mathrm{int}}}} \\
S_{\text {LOFI }}\left(F_{e}, R_{e}\right) \approx R_{b s} \frac{2\left(1-R_{b s}\right) \sqrt{R_{e}}\left\langle p_{\text {out }}\right\rangle}{\sqrt{2}} \frac{\gamma_{c}}{2} \frac{1}{2 \pi\left|F_{e}-F_{R}\right|}
\end{gathered}
$$

and finally one obtains (far away from the resonance frequency), the following approximate expression of the LOFI SNR:

$$
\operatorname{SNR}\left(F_{e}, R_{e}, T_{\mathrm{int}}<<\tau_{R}\right) \approx \sqrt{R_{e}}\left(1-R_{b s}\right) \times \sqrt{R_{b s}\left\langle p_{\text {out }}\right\rangle T_{\mathrm{int}}} \sqrt{\frac{T_{\mathrm{int}}}{\tau_{R}}}
$$


A brief comparison of Eq. (18) and of Eq. (13) (which gives the LOFI shot noise limit), shows that for: $T_{\text {int }} \ll \tau_{R}$, the LOFI SNR is lower than the LOFI shot noise limit and becomes frequency independent when working very far away from the resonance frequency. In agreement with the theoretical predictions, Fig. 2(c) shows that the LOFI SNR (continuous line) saturates and therefore becomes frequency independent at very low and very high frequencies. One can also observe that in these conditions, the LOFI SNR is smaller than the corresponding LOFI shot noise limit (dash line) by a multiplicative factor given by: $\sqrt{\frac{T_{\mathrm{int}}}{\tau_{R}}}<1$.

Secondly: if we now work at resonance $\left(F_{e}=F_{R}\right)$, then the LOFI noise and the LOFI signal are given by:

$$
\begin{gathered}
N_{\text {Laser }}\left(F_{R}, T_{\text {int }}<<\tau_{R}\right) \approx 2 \sqrt{R_{b s}\left\langle p_{\text {out }}(t)\right\rangle} \frac{\gamma_{c} \tau_{R}}{2} \frac{1}{\sqrt{2 \tau_{R}}} \\
S_{\text {LOFI }}\left(F_{R}, R_{e}\right)=R_{b s} \frac{2\left(1-R_{b s}\right) \sqrt{R_{e}}\left\langle p_{\text {out }}\right\rangle}{\sqrt{2}} \frac{\gamma_{c} \tau_{R}}{2}
\end{gathered}
$$

and finally one easily obtains the resonant value of the LOFI SNR:

$$
\operatorname{SNR}\left(F_{R}, R_{e}, T_{\mathrm{int}} \ll<\tau_{R}\right)=\sqrt{R_{e}}\left(1-R_{b s}\right) \times \sqrt{R_{b s}\left\langle p_{\text {out }}\right\rangle \tau_{R}}=\sqrt{R_{e}}\left(1-R_{b s}\right) \times \sqrt{R_{b s}\left\langle p_{\text {out }}\right\rangle T_{\mathrm{int}}} \sqrt{\frac{\tau_{R}}{T_{\mathrm{int}}}}
$$

The middle part of Eq. (21) shows that the LOFI SNR is now independent from the integration time $\left(T_{\text {int }}\right)$. This surprising effect arises because the laser noise power spectrum [Eq. (8)] have a Lorentzian type profile leading to a limit noise value [See Eq. (20)] when the detection 
bandwidth is large compared to the resonance width $\left(\frac{1}{T_{\mathrm{int}}}>>\frac{1}{\tau_{R}}\right)$. A brief comparison of Eq. (21) and Eq. (13) shows that, for a laser with a slow response time $\left(T_{\mathrm{int}}<<\tau_{R}\right)$, the LOFI SNR at resonance is higher than the standard LOFI shot noise limit. In agreement with Eq. (21), Fig. 2(c) shows that the LOFI SNR (continuous line) at resonance is higher than the corresponding LOFI shot noise limit (dash line) by a multiplicative factor given by: $\sqrt{\frac{\tau_{R}}{T_{\mathrm{int}}}}>1$.

Thirdly, in the intermediate situation, where: $\frac{1}{T_{\mathrm{int}}}>>\left|\left(F_{e}-F_{R}\right)\right|>>\frac{1}{\tau_{R}}$, one can determine the frequency shift $\left(F_{e}\right)$ for which the LOFI SNR is equal to the LOFI shot noise limit. By equalizing Eqs. (12a) and (15) one obtains:

$$
\left|F_{e}-F_{R}\right| \approx \frac{1}{2 \pi \sqrt{\tau_{R} T_{\mathrm{int}}}}
$$

Finally, we conclude this section, by reminding that the stationary LOFI SNR $\left(S_{\text {LOFI }} / N_{\text {Laser }}\right)$ is frequency independent [see Fig. 2(a)] and above all shot noise limited for a laser with fast response time $\left(T_{\mathrm{int}}>>\tau_{R}\right)$. On the other hand, for a laser with slow response time $\left(T_{\mathrm{int}} \ll<\tau_{R}\right)$, the stationary LOFI SNR is frequency dependent [Fig. 2(c)], larger than the LOFI shot-noise limit near the relaxation frequency (i.e. when: $\left|F_{e}-F_{R}\right|<\frac{1}{2 \pi \sqrt{\tau_{R} T_{\text {int }}}}$ ) and smaller than the LOFI shot-noise limit far way from the relaxation frequency (i.e. when: $\left|F_{e}-F_{R}\right|>\frac{1}{2 \pi \sqrt{\tau_{R} T_{\mathrm{int}}}}$ ). More precisely the LOFI SNR is larger (by a factor given by $\sqrt{\frac{\tau_{R}}{T_{\mathrm{int}}}}>1$ ) than the LOFI shot noise limit 
when working at the resonance frequency and smaller (by a factor given by $\sqrt{\frac{T_{\text {int }}}{\tau_{R}}}<1$ ) when working very far away from the resonance frequency.

\section{Numerical simulation of the stationary LOFI SNR}

By using a Runge-Kutta method, we have numerically solved the set of differential equations (1) to determine the stationary LOFI SNR for different experimental conditions (i.e. for different values of $T_{\text {int }}$ compared to $\tau_{R}$ ). More precisely, we have compared the dynamical behavior of two lasers having the same output power $\left(\left\langle p_{\text {out }}\right\rangle\right)$ and the same relaxation frequency $\left(F_{R}\right)$, but having two different values of the LOFI gain $\left(G\left(F_{R}\right)=\gamma_{c} \tau_{R} / 2\right)$ induced by two different values of the laser response time $\left(\tau_{R}\right)$. Here, our aim is to determine the best laser for high quality imaging (i.e. large LOFI SNR).

Fig. 3 shows a comparison of the stationary LOFI SNR obtained with the two lasers, for different values of the shift frequency $\left(F_{e}\right)$ and for different values of the lock-in integration time $\left(T_{\text {int }}\right)$. For each experimental condition $\left(F_{e}, T_{\text {int }}\right)$, the stationary LOFI signal (obtained with $R_{e}=1 \times 10^{-10}$ ) and the LOFI noise (obtained with $R_{e}=0$ ) have been determined from an average of 100 measurements each (no scanning occurs) to reduce the SNR uncertainty.

For the laser having the shortest time response time $\left(\tau_{R}=4 \mu \mathrm{s}\right)$ and therefore the lowest value of the LOFI gain $\left(G\left(F_{R}\right)=1 \times 10^{4}\right)$, the numerical results shown on Fig. 3(a) are in good agreement with the analytical predictions given by Eq. (13). Indeed, using a laser with a fast response time $\left(T_{\text {int }}>\tau_{R}\right)$, the simulated LOFI SNR is almost frequency independent and the numerical 
simulations show values that are very close to the analytical predictions. Indeed, by using Eq. (13), with a laser output power of $P_{\text {out }}=60 \mathrm{~mW}$, a target effective reflectivity of $R_{e}=4 \times 10^{-10}$ and a beam splitter reflectivity $R_{b s}=0.5$ one obtains for the LOFI SNR the values given in Table 1, which correspond to the LOFI shot noise limit. A closer look at the results of Fig. 3(a) shows a small frequency dependence which seems to be more important for the shortest integration time. This small frequency dependence is also in agreement with the theoretical prediction [see Fig. 2(b) for comparison].

For the laser having the longest time response $\left(\tau_{R}=333 \mu \mathrm{s}\right)$ and therefore the highest value of the LOFI gain $\left(G\left(F_{R}\right)=8 \times 10^{5}\right)$, the results of the numerical simulations shown on Fig. 3(b) are now frequency dependent and much more complicated to analyze.

Firstly, using Eq. (6), with a LOFI gain $G\left(F_{R}\right)=8 \times 10^{5}$ and a beam splitter reflectivity $R_{b s}=0.5, \quad$ one $\quad$ obtains $\quad R_{\text {sat }}\left(F_{R}\right)=1.5 \times 10^{-12}<<R_{e}=4 \times 10^{-10} \quad$ and $R_{\text {sat }}\left(1.1 \times F_{R}\right)=8 \times 10^{-9}>R_{e}=4 \times 10^{-10}$. The LOFI signal is therefore saturated at the relaxation frequency $F_{R}$ and non-saturated for shift frequencies with: $F_{e} \geq 1.1 \times F_{R}$.

Secondly, the LOFI SNR shows an anti-resonance phenomenon for the longest integration times and a resonance phenomenon for the shortest integration times. In Fig. 3(b), the anti-resonance phenomenon observed for $T_{\text {int }}=200 \mu \mathrm{s}$ and $T_{\text {int }}=600 \mu \mathrm{s}$ comes simply from the saturation of the LOFI signal. The SNR resonance phenomenon observed for $T_{\mathrm{int}}=6 \mu s=0.018 \tau_{R}$ and $T_{\text {int }}=20 \mu s=0.06 \tau_{R}$ can be explained by the analytical results shown on Fig. 2(c), with nevertheless a resonance height less important due to the saturation of the LOFI signal. 
Finally for $F_{e} \geq 1.1 \times F_{R}$ and $T_{\mathrm{int}}<\tau_{R}$, the following condition is satisfied: $F_{e}-F_{R}>1 / T_{\mathrm{int}}$ and in agreement with Eq. (18) one can observe on Fig.3(b) that the LOFI SNR is always smaller (by a factor approximately given by $\sqrt{\frac{T_{\text {int }}}{\tau_{R}}}<1$ ) than the LOFI shot noise limit values given in Table 1 .
a) $G\left(F_{R}\right)=1 \times 10^{4} \quad \tau_{R}=4 \mu s$

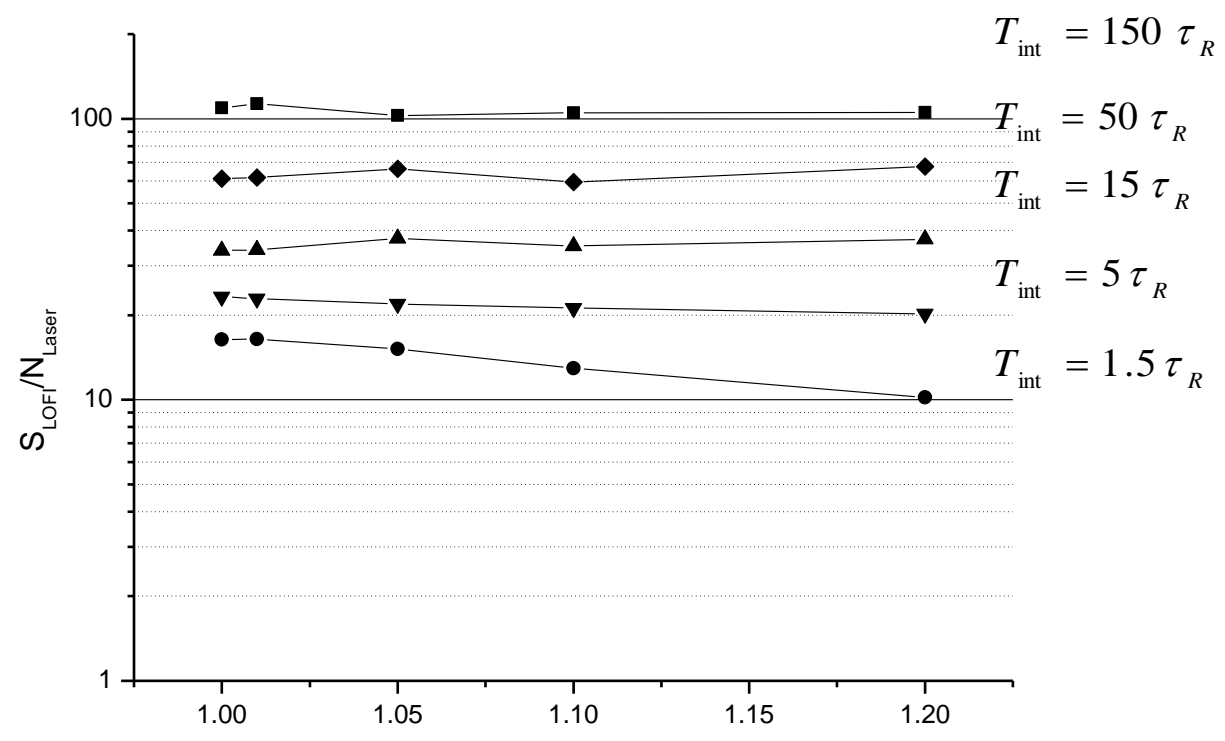

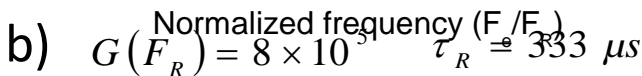

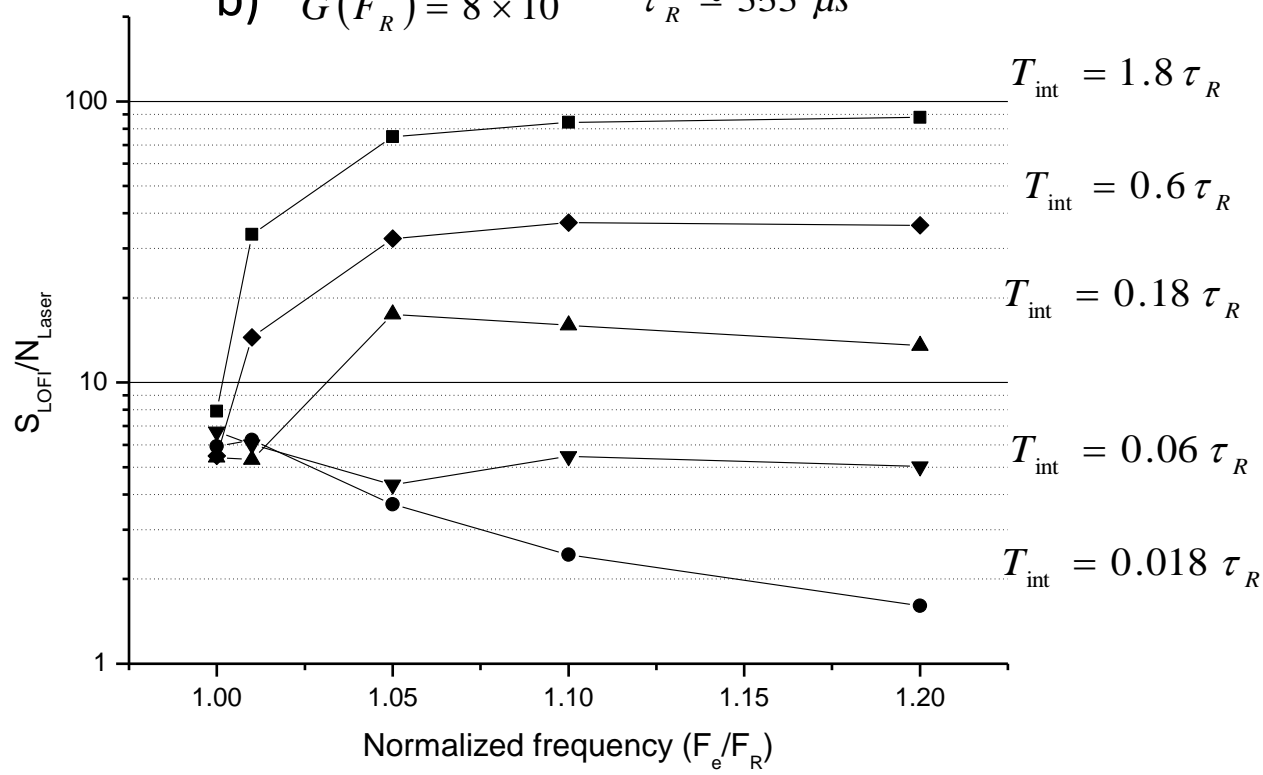

Fig. 3. SNR ( $S_{\text {LOFI }} / N_{\text {Laser }}$ ) of a class-B laser ( $P_{\text {out }}=60 \mathrm{~mW} ; F_{R}=356 \mathrm{kHz}, R_{e}=4 \times 10^{-10} R_{b s}=0.5$ ) versus the normalized shift-frequency $\left(F_{e} / F_{R}\right)$ for different values of the lock-in integration time: 
$(\mathbb{\square}) T_{\mathrm{int}}=600 \mu \mathrm{s},(\bullet) T_{\mathrm{int}}=200 \mu \mathrm{s},(\mathbf{\Delta}) T_{\mathrm{int}}=60 \mu \mathrm{s},(\boldsymbol{\nabla}) T_{\mathrm{int}}=20 \mu \mathrm{s},(\bullet) T_{\mathrm{int}}=6 \mu \mathrm{s}$. Upper graph $G\left(F_{R}\right)=1 \times 10^{4}$ and $\tau_{R}=4 \mu s \quad\left(\gamma_{c} / \gamma_{1}=10^{4}, r=1.002\right)$; Lower graph: $G\left(F_{R}\right)=8 \times 10^{5}$ and $\tau_{R}=333 \mu s \quad\left(\gamma_{c} / \gamma_{1}=10^{6}, r=1.2\right)$.

\begin{tabular}{|l|c|c|c|c|c|}
\hline$T_{\text {int }}$ & $6 \mu \mathrm{s}$ & $20 \mu \mathrm{s}$ & $60 \mu \mathrm{s}$ & $200 \mu \mathrm{s}$ & $600 \mu \mathrm{s}$ \\
\hline SNR & 9.7 & 17.7 & 30.6 & 55.9 & 96.8 \\
\hline
\end{tabular}

Table 1. LOFI SNR [Eq. (13)] obtained with the laser output power $P_{\text {out }}=60 \mathrm{~mW}$ (i.e. $p_{\text {out }}=3.2 \times 10^{17}$ photons $/ s$ at $\lambda=1064 \mathrm{~nm}$ ), a target effective reflectivity $R_{e}=4 \times 10^{-10}$ and a beam splitter reflectivity $R_{b s}=0.5$.

\section{AUTODYNE IMAGING}

\section{A. Transient LOFI signal}

Suppose now that due to the scanning of the laser beam on the target under investigation, the target properties suddenly change at a time $\mathrm{t}=0$ (for example at the edge of the target) with: $r_{e, 1}=\sqrt{R_{e, 1}} \exp \left(j \phi_{e, 1}\right)$ for $t<0$ and $r_{e, 2}=\sqrt{R_{e, 2}} \exp \left(j \phi_{e, 2}\right)$ for $t \geq 0$. Under these conditions, the laser output power modulation is composed of a stationary signal, for $t<0$ :

$$
p_{\text {out }}\left(t<0, F_{e}, r_{e, 1}\right)=\left\langle p_{\text {out }}\right\rangle+2 G\left(F_{e}\right)\left(1-R_{b s}\right) \sqrt{R_{e, 1}}\left\langle p_{\text {out }}\right\rangle \cos \left(2 \pi F_{e} t+\Phi_{e, 1}\right)
$$

and for $t \geq 0$, of the sum of a stationary and of a transient signal:

$$
\begin{aligned}
p_{\text {out }}\left(t \geq 0, F_{e}, r_{e, 2}\right)= & \left\langle p_{\text {out }}\right\rangle+2 G\left(F_{e}\right)\left(1-R_{b s}\right) \sqrt{R_{e, 2}}\left\langle p_{\text {out }}\right\rangle \cos \left(2 \pi F_{e} t+\Phi_{e, 2}\right) \\
& +\exp \left(-\frac{t}{\tau_{R}}\right) 2 G\left(F_{e}\right)\left(1-R_{\text {bs }}\right) \sqrt{C}\left\langle p_{\text {out }}\right\rangle \cos \left(2 \pi F_{R} t+\Phi_{c}\right)
\end{aligned}
$$


where for a class-B laser $\left(F_{R}>>1 / \tau_{R}\right)$, we assume that the transient signal is an oscillating signal centered at the relaxation frequency $F_{R}$. The constants $C$ and $\Phi_{c}$ can be determined by ensuring the continuity of the signal and of its first derivative.

$$
\begin{gathered}
\sqrt{R_{e, 1}} \cos \left(\Phi_{e, 1}\right)=\sqrt{R_{e, 2}} \cos \left(\Phi_{e, 2}\right)+\sqrt{C} \cos \left(\Phi_{c}\right) \\
\sqrt{R_{e, 1}} \frac{F_{e}}{F_{R}} \sin \left(\Phi_{e, 1}\right)=\sqrt{R_{e, 2}} \frac{F_{e}}{F_{R}} \sin \left(\Phi_{e, 2}\right)+\sqrt{C} \sin \left(\Phi_{c}\right)-\frac{1}{2 \pi \tau_{R} F_{R}} \sqrt{C} \cos \left(\Phi_{c}\right)
\end{gathered}
$$

For a class-B laser $\left(F_{R}>>1 / \tau_{R}\right)$, Eqs. (24) shows that, whatever the experimental conditions, a good approximation of the amplitude of the transient oscillations (i.e. the order of magnitude of $C$ ) is given by:

$$
C \approx R_{e, 1}+R_{e, 2}-2 \sqrt{R_{e, 1}} \sqrt{R_{e, 2}} \cos \left(\Phi_{e, 2}-\Phi_{e, 1}\right)
$$

and therefore

$$
\left|R_{e, 2}-R_{e, 1}\right| \leq C \leq\left|R_{e, 2}+R_{e, 1}\right|
$$

If $R_{e, 1}<<R_{e, 2}$, Eqs. (25) show that the amplitude of the transient signal $(\propto \sqrt{C}$ ) and of the stationary signal $\left(\propto \sqrt{R_{e, 2}}\right)$ are approximately equal $\left(C \approx R_{e, 2}\right)$, while in the contrary case $R_{e, 1}>R_{e, 2}$, the transient signal is higher than the stationary signal $\sqrt{C} \approx \sqrt{R_{e, 1}}>\sqrt{R_{e, 2}}$. 
In the most general case, $R_{e, 1}$ and $R_{e, 2}$ are of the same order of magnitude $\left(R_{e, 1} \approx R_{e, 2}\right)$ and the transient signal is at maximum of the same order of magnitude than the stationary signal $\left(0 \leq C \leq 2 R_{e, 2}\right)$.

At the output of the lock-in amplifier, using an integration time $T_{\text {int }}$ and a first order filter, the transient LOFI signal at the demodulation frequency $F_{e}$ can be express in the time domain by:

$$
T_{\text {LOFI }}\left(F_{e}, C, T_{\mathrm{int}}\right)=\left|\frac{1}{T_{\mathrm{int}}} \int_{0}^{+\infty} \exp \left(-\frac{t}{T_{\mathrm{int}}}\right) R_{b s} \sqrt{2} 2 G\left(F_{e}\right)\left(1-R_{b s}\right) \sqrt{C}\left\langle p_{\text {out }}\right\rangle T_{R}(t) \exp \left(-j 2 \pi F_{e} t\right) d t\right|(26)
$$

with the transient shape: $T_{R}(t)=\exp \left(-\frac{t}{\tau_{R}}\right) \cos \left(\Omega_{R} t+\Phi_{c}\right)$, and where the averaging shape $\frac{1}{T_{\mathrm{int}}} \exp \left(-\frac{t}{T_{\mathrm{int}}}\right)$ corresponds simply to the Fourier Transform of the first order filter $F_{\mathrm{int}}\left(F, T_{\mathrm{int}}\right)$ defined previously by Eq. (9b).

For $T_{\text {int }} F_{e} \gg 1$ (i.e. no frequency mismatch), one obtains:

$$
T_{\text {LOFI }}\left(F_{e}, C, T_{\text {int }}\right)=R_{b s} \frac{2 G\left(F_{e}\right)\left(1-R_{b s}\right) \sqrt{C}\left\langle p_{\text {out }}\right\rangle}{\sqrt{2}} \frac{1}{T_{\text {int }}} \frac{1}{\sqrt{\left(\frac{1}{T_{\text {int }}}+\frac{1}{\tau_{R}}\right)^{2}+\left(2 \pi F_{e}-2 \pi F_{R}\right)^{2}}} .
$$

or identically by introducing the LOFI signal given by Eq. (7):

$$
T_{\text {LOFI }}\left(F_{e}, C, T_{\mathrm{int}}\right)=S_{\text {LOFI }}\left(F_{e}, R_{e, 2}\right) \sqrt{\frac{C}{R_{e, 2}}} \frac{1}{T_{\mathrm{int}}} \frac{1}{\sqrt{\left(\frac{1}{T_{\mathrm{int}}}+\frac{1}{\tau_{R}}\right)^{2}+\left(2 \pi F_{e}-2 \pi F_{R}\right)^{2}}}
$$


Fig. 4 shows the normalized $\left(\sqrt{R_{e, 2} / C}=1\right)$ ratio between the stationary and the transient LOFI signals $\left(S_{\text {LOFI }} / T_{\text {LOFI }}\right)$ versus the normalized shift frequency $\left(F_{e} / F_{R}\right)$ for different values of the lock-in integration time $\left(T_{\text {int }}\right)$ compared to the transient time $\left(\tau_{R}\right)$.

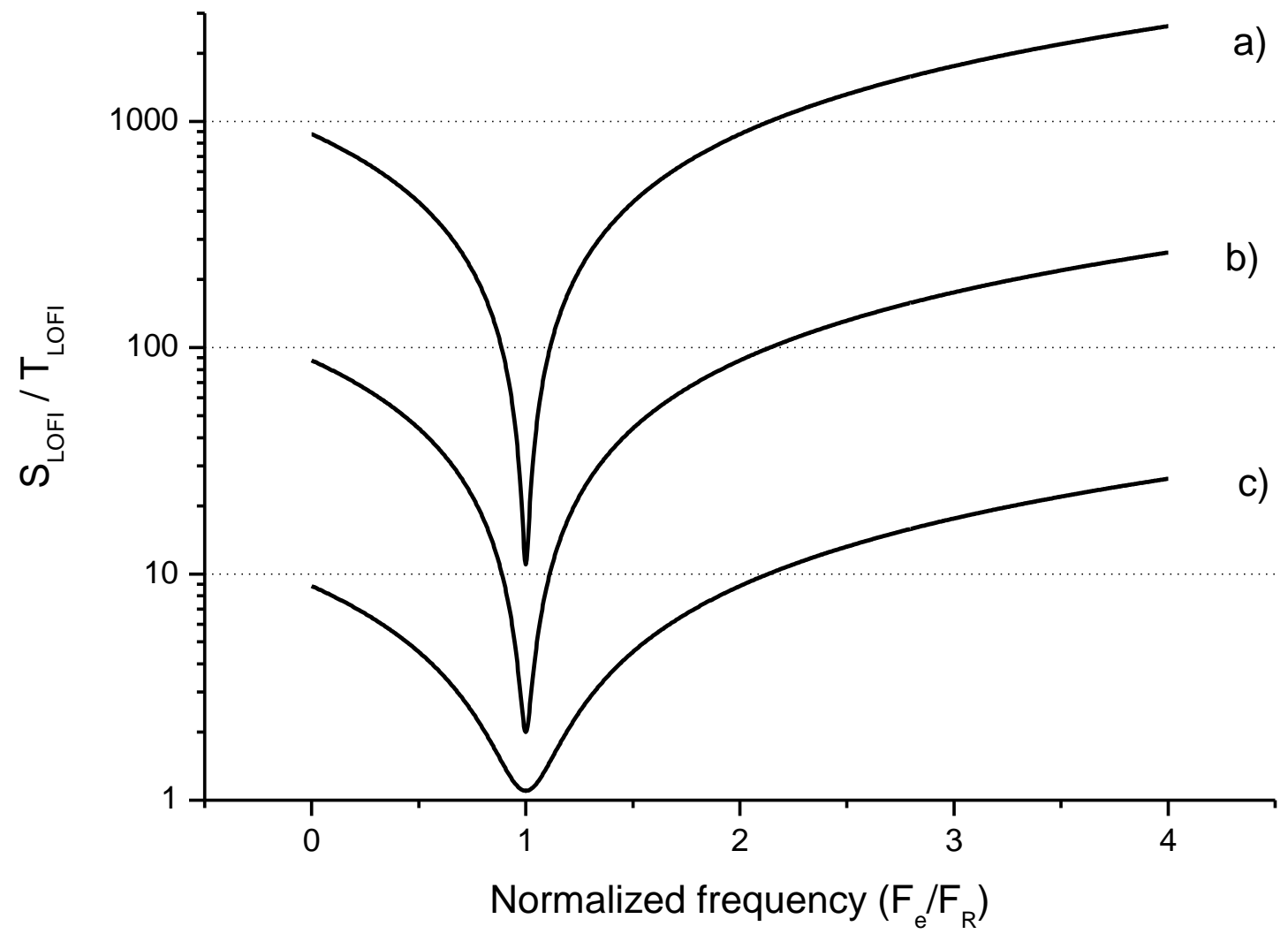

Fig. 4. Normalized $\left(R_{e, 2} / C=1\right)$ ratio between the stationary and the transient LOFI signals ( $S_{\text {LOFI }} / T_{\text {LOFI }}$ ) versus the normalized shift frequency $\left(F_{e} / F_{R}\right)$ for different values of the lock-in integration time $\left(T_{\text {int }}\right)$ compared to the transient time $\left(\tau_{R}\right):$ a) $T_{\text {int }}=10 \times \tau_{R}$, b) $T_{\text {int }}=\tau_{R}$ c) $T_{\text {int }}=\tau_{R} / 10$. The laser is a class-B laser with $F_{R} \tau_{R} \approx 14$.

B. Imaging with a fast response time laser $\left(T_{\mathrm{int}} \gg \tau_{R}\right)$ 
With a fast response time laser and with $C \approx R_{e, 2}$ (corresponding to the most general case), Eq. (27b) gives:

$$
S_{\text {LOFI }}\left(F_{e}, R_{e, 2}\right) \approx T_{\text {LOFI }}\left(F_{e}, C, T_{\mathrm{int}}>>\tau_{R}\right) \frac{T_{\mathrm{int}}}{\tau_{R}} \sqrt{1+\left(2 \pi F_{e}-2 \pi F_{R}\right)^{2} \tau_{R}^{2}}
$$

which can be reduce to the following inequality:

$$
S_{\text {LOFI }}\left(F_{e}, R_{e, 2}\right) \gg>T_{\text {LOFI }}\left(F_{e}, C, T_{\text {int }} \gg \tau_{R}\right)
$$

Fig. 4(a) confirms that by using a laser with a fast response time, the stationary LOFI signal is generally higher than the transient LOFI signal, whatever the working frequency $F_{e}$ is.

\section{Imaging with a slow response time laser $\left(T_{\mathrm{itt}}<\tau_{R}\right)$}

With a slow response time laser and with $C \approx R_{e, 2}$ (corresponding to the most general case), Eq. (27b) becomes:

$$
S_{\text {LOFI }}\left(F_{e}, R_{e, 2}\right) \approx T_{\text {LOFI }}\left(F_{e}, C, T_{\mathrm{int}}<<\tau_{R}\right) \sqrt{1+\left(2 \pi F_{e}-2 \pi F_{R}\right)^{2} T_{\mathrm{int}}^{2}},
$$

and two different cases need to be analyzed. Firstly, if we work near the resonance frequency,

with $\left|F_{e}-F_{R}\right|<<\frac{1}{T_{\text {int }}}$, Eq. (30) reduces to:

$$
S_{\text {LOFI }}\left(F_{e} \approx F_{R}, R_{e, 2}\right) \approx T_{\text {LOFI }}\left(F_{e} \approx F_{R}, C, T_{\mathrm{int}}<<\tau_{R}\right)
$$


Fig. 4(c) confirms that by using a laser with a slow response time, the stationary LOFI signal and the transient signal are of the same order of magnitude near the relaxation frequency:

Secondly, if we work far away from the resonance frequency, $\left(\left|F_{e}-F_{R}\right|>>\frac{1}{T_{\mathrm{int}}}\right)$, Eq. (30) gives:

$$
S_{\text {LOFI }}\left(F_{e}, R_{e, 2}\right)>>T_{\text {LOFI }}\left(F_{e}, C, T_{\text {int }}<<\tau_{R}\right)
$$

and the transient dynamics can be ignored compared to the stationary dynamics.

Fig. 4(c) confirms that by using a laser with a slow response time, the stationary LOFI signal is higher than the transient signal when working far way the relaxation frequency

At this point one can also notice that by using a laser with a slow response time $\left(T_{\text {int }}<<\tau_{R}\right)$, the minimization of the transient signal far away from the resonance is made to the detriment of the LOFI gain. Indeed for $F_{e}>>F_{R}+\frac{1}{T_{\mathrm{int}}}>>F_{R}+\frac{1}{\tau_{R}}$, the value of usable LOFI gain [Eq. (3)] is limited by the following inequality:

$$
G\left(F_{e}\right)<<\frac{T_{\text {int }}}{\tau_{R}} G\left(F_{R}\right)
$$

Finally, we conclude this section by reminding that the transient signal can be neglected compared to the stationary signal if one uses a laser with a fast response time $\left(T_{\text {int }}>>\tau_{R}\right)$ whatever the working frequency is, and also with slow response time laser $\left(T_{\mathrm{int}}<<\tau_{R}\right)$ if the working frequency is far away enough from the relaxation frequency $\left(\left|F_{e}-F_{R}\right|>>\frac{1}{T_{\text {int }}}\right)$. In a previous experimental paper [19] we have shown that the best working frequency called $F_{e,+}$ is 
the frequency at which the laser quantum noise is equal to the detector noise level. In the mentioned paper, the laser time response is $\tau_{R} \approx 20 \mu \mathrm{s}$, the laser relaxation frequency is $F_{R}=1.8 \mathrm{MHz}$ and we have experimentally determined $F_{e,+} \approx 6 \mathrm{MHz}$. Under these conditions, the transient dynamics can be neglected if the following inequality is verified:

$T_{\mathrm{int}}>\frac{1}{\left|F_{e,+}-F_{R}\right|} \approx 0.2 \mu \mathrm{s}$. Therefore one can use $T_{\mathrm{int}}=2 \mu \mathrm{s} \approx \frac{\tau_{R}}{10}$ to obtain high speed LOFI imaging without any perturbation from the transient signal $\left(S_{\text {LOFI }}\left(F_{e+}, R_{e, 2}\right) / T_{\text {LOFI }}\left(F_{e+}, R_{e, 2}, T_{\text {int }}\right) \approx 53\right)$. Note also that these experimental conditions are also compatible with our starting hypothesis $\left(T_{\text {int }} F_{e+}=12>>1\right)$.

\section{Numerical simulation}

In the most general situation, the LOFI SNR is given by the ratio between the LOFI signal divided by the detector noise plus the laser quantum noise plus the transient fluctuation. Here, our main objective is to compare the laser quantum noise with the transient fluctuations which can become important for short integration times (i.e. fast imaging condition). We have therefore neglected the detector noise in the current numerical study.

To show the effect of the transient dynamics on LOFI imaging, we have compared 1D scans extracted from the measured output power modulation. These scans have been obtained from the numerical integration of the set of Eqs. (1) (with no averaging). The target under investigation is a symmetric reflectivity pyramid composed of four levels which allows the observation of the transient dynamics effects during the scan in the case of an effective reflectivity increase $\left(R_{e, 1}<<R_{e, 2}\right)$ or decrease $\left(R_{e, 1}>>R_{e, 2}\right)$. For the current numerical study, we have chosen very low values of the effective reflectivity to study the LOFI sensitivity under 
ultimate conditions where the transient dynamics can be of the same order of magnitude than the laser quantum noise. Fig. 5 shows the numerical results obtained with the two lasers already studied in the section 3 of the present manuscript. To visually separate the transient dynamics effects from the noise effects on the LOFI images, the solid curves are numerically realized without laser quantum noise (i.e. without the Langevin noise forces), while the curves with circles combine both effects (transient signal and quantum noise).

a) $\quad F_{e}=F_{R}, \quad T_{\mathrm{int}}=20 \mu s=5 \tau_{R}$

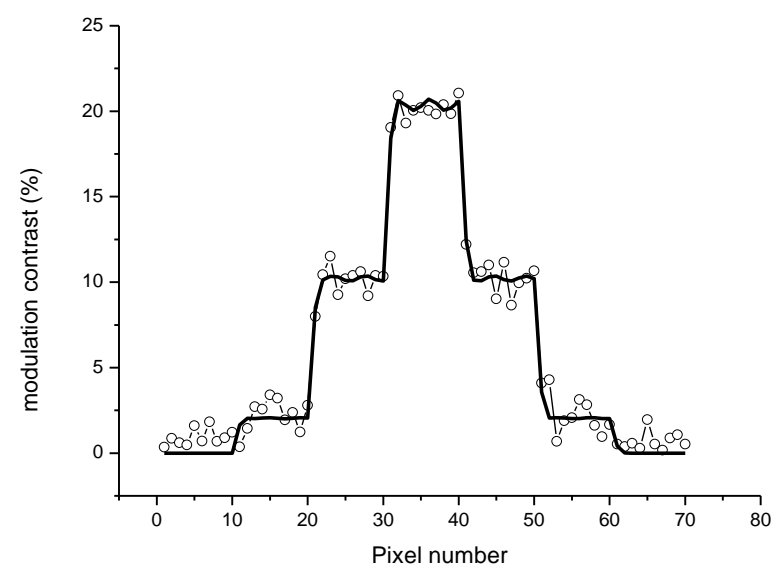

c) $F_{e}=F_{R}, \quad T_{\text {int }}=20 \mu s=0.06 \tau_{R}$

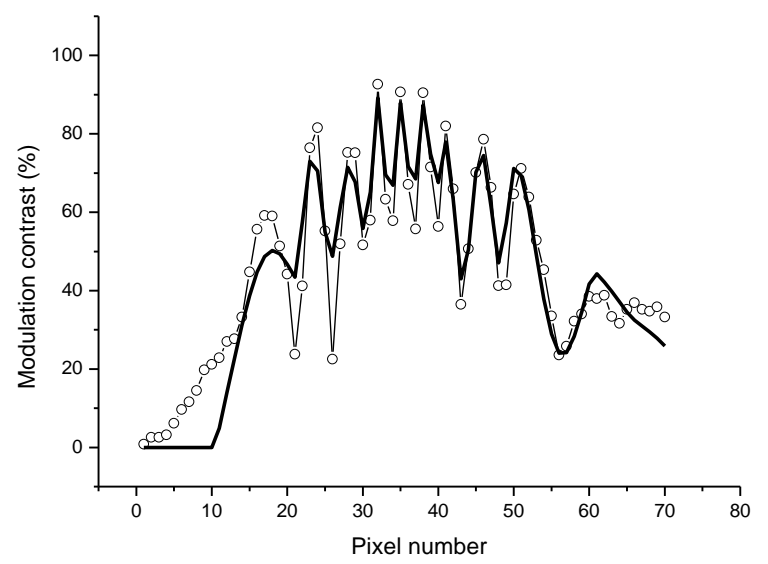

b) $\quad F_{e}=1.5 \times F_{R}, \quad T_{\mathrm{itt}}=20 \mu s=5 \tau_{R}$

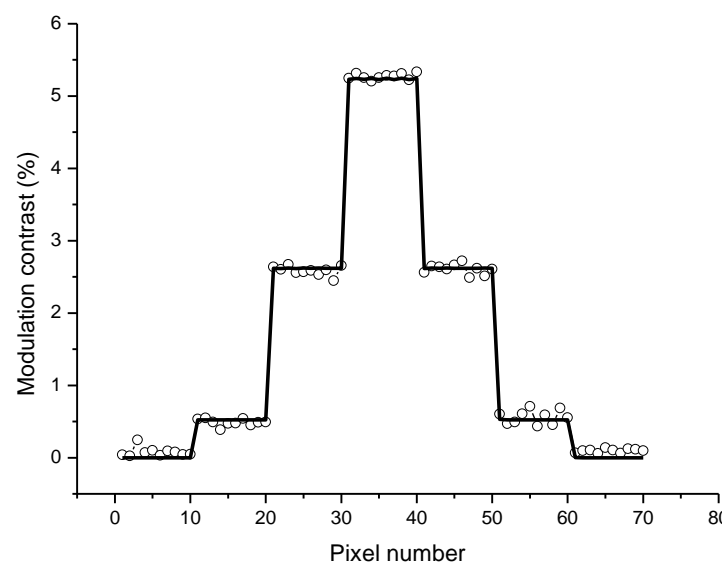

d) $\quad F_{e}=1.5 \times F_{R}, \quad T_{\mathrm{int}}=20 \mu s=0.06 \tau_{R}$

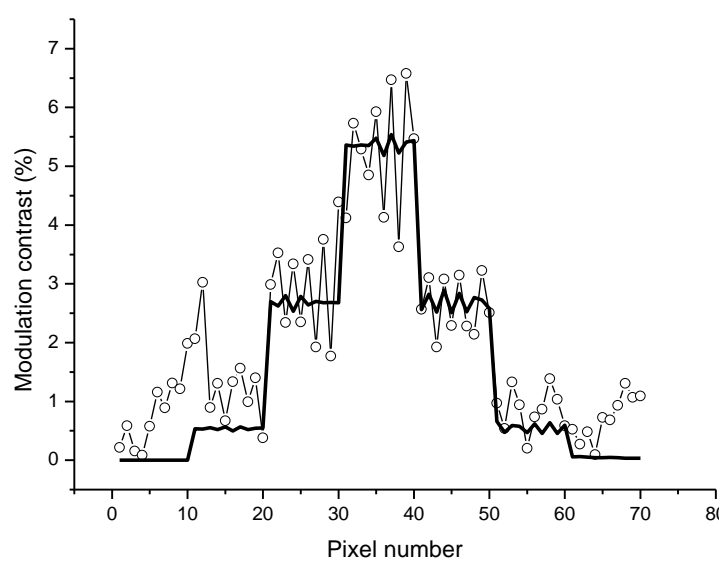

Fig. 5. Numerical 1D scan obtained from the measured laser output power MC of a LOFI set-up, when the laser is scanned on a symmetric reflectivity pyramid composed of 4 levels. Experimental conditions: $P_{\text {out }}=60 \mathrm{~mW}$ (i.e. $p_{\text {out }}=3.2 \times 10^{17}$ photons $/ \mathrm{s}$ at $\lambda=1064 \mathrm{~nm}$ ), $F_{R}=356 \mathrm{kHz}, T_{\text {int }}=20 \mu \mathrm{s}$. Level 1: (pixels 1-10 \& 61-70), $R_{e}=0$; level 2: (pixels 11-20 \& 51-60), $R_{e}=4 \times 10^{-12}$; level 3: (pixels 21-30 \& 41- 
50), $R_{e}=1 \times 10^{-10}$; level 4: (pixels 31-40), $R_{e}=4 \times 10^{-10}$. Top row: $G\left(F_{R}\right)=1 \times 10^{4}$ with $\tau_{R}=4 \mu \mathrm{s}$; bottom row: $G\left(F_{R}\right)=8 \times 10^{5}$ with $\tau_{R}=330 \mu \mathrm{s}$; left column: $F_{e}=F_{R}$; right column: $F_{e}=1.5 \times F_{R}$. Curves with circles $(O)$ : results with laser quantum noise; Solid curves $(-)$ : results without laser quantum noise.

For the laser having the lowest value of the LOFI gain $\left(G\left(F_{R}\right)=1 \times 10^{4}\right)$, i.e. the shortest response time $\left(\tau_{R}=4 \mu s\right)$, the numerical results shown on Figs. 5(a) and 5(b) are in good agreement with the analytical predictions of the section 3. Indeed, the transient dynamics fluctuations are always smaller than the laser quantum noise fluctuations, which confirms the fact that by using a laser with a fast response time $\left(T_{\mathrm{int}}=20 \mu s>\tau_{R}\right)$, the transient dynamics is negligible whatever the working frequency is. Note also that when the effective reflectivity is multiplied by a factor 100 (level $\mathrm{n}^{\circ} 2$ to level $\mathrm{n}^{\circ} 4$ ), the MC increases by a factor 10 , while when the effective reflectivity is multiplied by a factor 4 (level $n^{\circ} 3$ to level $n^{\circ} 4$ ) the MC increases by a factor 2 .

Moreover, in Table 2, the MC and the SNR, numerically determined using the curves with the circles of Figs. 5(a) and 5(b) and analytically calculated [from Eqs. (5) and (11)], are very close. One can notice that the good agreement comes from the fact that for this laser, the optical feedback level is below the saturation level of the laser $\left(R_{e, 4}=4 \times 10^{-10}<R_{\text {sat }}\left(F_{R}\right)=1 \times 10^{-8}\right)$

and because the transient fluctuations are negligible in the amount of noise. With this laser, one also observes that by increasing the shift frequency, the values of the $\mathrm{MC}$ is smaller but that the SNR remains approximately unchanged, which confirms again the fact that by using a laser with a fast time response $\left(T_{\text {int }}>\tau_{R}\right)$ the LOFI SNR is approximately frequency independent, in agreement with Eq. (13). 


\begin{tabular}{|l|l|l|l|l|}
\hline $\mathrm{R}_{\mathrm{e}}$ & 0 & $4 \times 10^{-12}$ & $1 \times 10^{-10}$ & $4 \times 10^{-10}$ \\
\hline$F_{e}=F_{R}$ & & $\mathbf{2 . 2}(2.0)$ & $\mathbf{1 0 . 2}(10.2)$ & $\mathbf{2 0 . 1}(20.5)$ \\
$\mathrm{MC}(\%)$ & $\mathbf{1}(1)$ & $\mathbf{2 . 1}(1.9)$ & $\mathbf{1 0 . 2}(9.7)$ & $\mathbf{2 4 . 1}(19.4)$ \\
$\mathrm{SNR}$ & & & & \\
\hline$F_{e}=1.5 \times F_{R}$ & $\mathbf{0 . 2}(0)$ & $\mathbf{0 . 6}(0.5)$ & $\mathbf{2 . 7}(2.6)$ & $\mathbf{5 . 2}(5.2)$ \\
$\mathrm{MC}(\%)$ & $\mathbf{1}(1)$ & $\mathbf{2 . 5}(1.6)$ & $\mathbf{1 0 . 1}(8.2)$ & $\mathbf{1 8 . 7}(16.3)$ \\
$\mathrm{SNR}$ & &
\end{tabular}

Table 2. MC and SNR of the LOFI images [Figs. 5(a) and 5(b)] obtained with the laser having the lower value LOFI gain $\left(\mathrm{G}\left(\mathrm{F}_{\mathrm{R}}\right)=1 \times 10^{4}\right)$. Numerical results are in bold while the analytical results (Eq.11) are written in italics between parentheses. In the numerical results the noise is composed of the transient fluctuation plus the laser quantum noise while in the analytical results, the noise corresponds only to the laser quantum noise.

\begin{tabular}{|c|c|c|c|c|}
\hline $\mathrm{R}_{\mathrm{e}}$ & 0 & $4 \times 10^{-12}$ & $1 \times 10^{-10}$ & $4 \times 10^{-10}$ \\
\hline $\begin{array}{l}F_{e}=F_{R} \\
\mathrm{MC}(\%) \\
\mathrm{SNR}\end{array}$ & $\begin{array}{l}22.2(0) \\
\mathbf{1}(1)\end{array}$ & $\begin{array}{l}\mathbf{4 2 . 3}(166.7) \\
\mathbf{2 . 8}(7.4)\end{array}$ & $\begin{array}{l}\mathbf{5 7 . 6}(833.3) \\
\mathbf{3 . 1}(37.2)\end{array}$ & $\begin{array}{l}\mathbf{7 0 . 4}(1667.0) \\
\mathbf{4 . 6}(74.3)\end{array}$ \\
\hline $\begin{array}{l}F_{e}=1.5 \times F_{R} \\
\operatorname{MC}(\%) \\
\operatorname{SNR}\end{array}$ & $\begin{array}{l}\mathbf{0 . 8}(0) \\
\mathbf{1}(1)\end{array}$ & $\begin{array}{l}\mathbf{1 . 1}(0.5) \\
\mathbf{2}(0.4)\end{array}$ & $\begin{array}{l}2.8(2.7) \\
4(2.1)\end{array}$ & $\begin{array}{l}5.2(5.4) \\
5(4.2)\end{array}$ \\
\hline
\end{tabular}

Table 3. MC and SNR of the LOFI images [Figs. 5(c) and 5(d)] obtained with the laser having the higher value LOFI gain $\left(G\left(F_{R}\right)=8 \times 10^{5}\right)$. Numerical results are in bold while the analytical results [Eq.(11)] are written in italics between parentheses. In the numerical results the noise is composed of the transient fluctuation plus the laser quantum noise while in the analytical results, the noise corresponds only to the laser quantum noise.

Now, if the value of the LOFI gain $\left(G\left(F_{R}\right)=8 \times 10^{5}\right)$ is increased, by increasing the laser response time $\left(\tau_{R}=330 \mu s\right)$, one can observe [compare Figs. 5(c) and 5(a)] that the MC is higher but that the SNR is lower with the laser having the highest LOFI gain (i.e. the longest response time). The degradation of the SNR comes from the high value of the transient LOFI signal [see Eq. (31)], characterized on Fig. 5(c) by transient dynamic fluctuations as high as the the laser quantum noise fluctuations. The degradation of the LOFI SNR also comes from the saturation of the LOFI signal of this oversensitive laser 
$\left(R_{\text {sat }}\left(F_{R}\right)=1.5 \times 10^{-12}<R_{e} / 100=4 \times 10^{-12}\right)$. The saturation effect is also visible in Table 3 where for both SNR and MC, the numerical results [obtained from Fig. 5(c)] are always lower than the analytical ones, obtained from Eq. (5) for the MC and from Eq. (11) for the calculation of the SNR [24].

At this point one can also notice that due to the very high value of the transient LOFI signal (linked to the slow response time of the laser: $T_{\text {int }}=0.06 \tau_{R}$ ) and due to the saturation of the LOFI signal (induced by the high value of the LOFI gain: $G\left(F_{R}\right)=8 \times 10^{5}$ ), the LOFI signal is hardly perturbed. In these conditions, the observation at the relaxation frequency $F_{e} \approx F_{R}$, of a LOFI SNR higher than the LOFI shot noise limit [see Eq. (21) and Fig. 2(c)] is unfortunately unobtainable (i.e. experimentally unobservable) in the imaging condition [25].

To decrease the transient dynamics effects and to avoid the laser dynamic saturation observed on Fig. 5(c), one needs to work far away from the resonance frequency. If we now compare Figs. 5(c) and 5(d), one can observe that for $F_{e}=1.5 \times F_{R}$ the MC is lower but that the LOFI SNR has increased (the pyramid is roughly distinguishable). In Fig. 5(d) one can also observe that the SNR is now limited by the laser quantum noise (circles fluctuations) which is higher than the LOFI transient fluctuations (solid curve fluctuations). In agreement with Eq. (25), one can also observe on Fig. 5(d) that the transient dynamics effect is much more important for a decreasing step $\left(R_{e, 1}>>R_{e, 2}\right)$ than for an increasing step $\left(R_{e, 1}<<R_{e, 2}\right)$ if we look at the solid curve. If we now look at the curve with the circle, one can also observed that this dissymmetric effect is completely hide by the laser quantum noise. 
Finally, the comparison of Figs 5(b) and 5(d) shows that far away from the resonance, the two lasers have approximately the same gain $\left(G\left(1.5 \times F_{R}\right) \approx 2 \times 10^{3}\right)$ and therefore the same MC [Eq. (5)]. One can also clearly observe that for the same integration time $\left(T_{\text {int }}=20 \mu \mathrm{s}\right)$, the best LOFI image (the best SNR) is clearly obtained when using the laser with the lowest response time allowing the following condition $\left(T_{\mathrm{int}}>\tau_{R}\right)$ and therefore a shot noise limited LOFI detection [see Eq. (13)].

Finally for fast imaging, we need a time value of $T_{\text {int }}$ as short as possible and to be shot noise limited we need to use a laser with a fast response time $\left(T_{\mathrm{int}}>\tau_{R}\right)$. For LOFI imaging, the best laser is therefore a class-B laser with the shortest possible value of $\tau_{R}$ allowing the use of relatively short integration time.

In practical LOFI experiments, to be shot noise limited, the laser quantum noise needs to be just above the detector noise. So finally, this is the detector noise level which determines the lowest possible value of the LOFI gain $[18,19]$ and therefore the shortest possible value of the laser response time. For this particular time, the laser used in the LOFI experiment is optimized and allows to obtain image as fast as possible with a shot noise limited detection. In a previous paper [18], we have shown that for a detection noise level characterized by a noise equivalent power: NEP $(W / \sqrt{H z}$ ). The optimum value for le LOFI gain is given by:

$$
G_{\text {opt }}\left(F_{R}\right)=\left(\frac{\gamma_{c}}{\gamma_{1} r}\right)_{\text {opt }}=\frac{1}{\sqrt{2 R_{b s}}} \frac{N E P /(h c / \lambda)}{\sqrt{\left\langle p_{\text {out }}\right\rangle}}
$$

which allows to determine the optimum value of the laser response time : 


$$
\tau_{R, o p t}=\left(\frac{2}{\gamma_{1} r}\right)_{o p t}=\frac{2}{\sqrt{2 R_{b s}}} \frac{N E P /(h c / \lambda)}{\gamma_{c} \sqrt{\left\langle p_{\text {out }}\right\rangle}}
$$

For example, for a laser with an output power $P_{\text {out }}=60 \mathrm{~mW} \quad\left(\left\langle p_{\text {out }}\right\rangle=3.2 \times 10^{17}\right.$ photons $/ \mathrm{s}$ at $\lambda=1064 \mathrm{~nm}$ ), a cavity damping rate $\gamma_{c}=5 \times 10^{9} \mathrm{~s}^{-1}$ and for a setup with a beam splitter reflectivity $\mathrm{R}_{\mathrm{bs}}=0.5$ and a noise equivalent power $N E P=6 \times 10^{-9} \mathrm{~W} / \sqrt{\mathrm{Hz}}$, one obtains: $G_{\text {opt }}\left(F_{R}\right) \approx 57$ and finally $\tau_{R, o p t} \approx 23 n s$.

Therefore we can take: $T_{\text {int }}=10 \times \tau_{R, o p t}=0.23 \mu \mathrm{s}$, as the minimum acquisition time compatible with the shot-noise condition $\left(T_{\text {int }}>>\tau_{R}\right)$. To be compatible with our initial hypothesis $\left(F_{R} T_{\text {int }}>>1\right)$, we also take a factor 10 and the laser relaxation frequency needs to as high as: $F_{R}=\frac{10}{T_{\mathrm{int}}}=\frac{1}{\tau_{R, \text { opt }}} \approx 40 \mathrm{MHz}$

Finally, for high speed imaging combined to shot-noise limited detection, a laser diode with:, $\tau_{R}=2 n s$ and $G\left(F_{R}\right)=5 \times 10^{2}\left(\gamma_{c}=5 \times 10^{11} s^{-1}, \gamma_{1}=5 \times 10^{8} s^{-1}, \mathrm{r}=2\right)$ seems to be an interesting candidate compared to microchip laser with $\tau_{R}=200 \mu s$ and $G\left(F_{R}\right)=5 \times 10^{5} \quad\left(\gamma_{c}=5 \times 10^{9} s^{-1}\right.$, $\left.\gamma_{1}=5 \times 10^{3} s^{-1}, \quad r=2\right)$. However the use of a laser diode with a relaxation frequency $F_{R}=2.5 \mathrm{GHz} \quad$ (compare to $F_{R}=800 \mathrm{kHz}$ for a solid state microchip laser) requires the use an electro-optics modulator to generate the frequency shift (instead of an acousto optics modulator) and above all requires a rapid electronic detection (with a gigahertz bandwidth) which is technically much more complicated to carry out than an electronic setup with a megahertz bandwidth. 
Even if a laser diode seems to be an interesting candidate for LOFI experiments, the theoretical and experimental study needs to be done to verify this possibility. Indeed the laser rate equations used in this paper are not completely correct to describe the dynamical behavior of a laser diode. Indeed, our modeling doesn't take into account of the phase amplitude coupling of the laser electric field (i.e. the Henry factor) occurring inside the laser cavity of a laser diode and also doesn't take into account of the optical feedback time delay which cannot be neglected for a laser diode with a laser relaxation frequency in the gigahertz range. Indeed, for $F_{R}=1 \mathrm{GHz}$ and for an optical feedback time delay $\tau=10 \mathrm{~ns}$ (which correspondst to laser-target distance of 1.5m), one obtained $2 \pi \times F_{R} \tau=2 \pi \times 10>>1$. Therefore even if the laser diode seems to be a good candidate, the comparison between a laser diode and a solid-state laser for autodyne interferometry cannot be made so directly and therefore needs to be made carefully.

\section{CONCLUSION}

In a LOFI setup, the beating between the reference beam and the signal beam takes place inside the laser cavity and therefore the laser fulfills simultaneously the roles of emitter and detector of photons. In these conditions, the laser relaxation oscillations play a leading role both in the laser quantum noise which determines the SNR and in the laser transient dynamics which determines the response time of the LOFI setup. In the present study, we have theoretically compared the stationary LOFI SNR and the LOFI response time of two lasers having the same output power, the same relaxation frequency, but having two different values of the LOFI gain induced by two different values of the laser response time. 
Firstly, we have determined that the stationary LOFI SNR is frequency independent and above all shot noise limited when the response time of the laser is shorter than the lock-in integration time $\left(\tau_{R}<<T_{\text {int }}\right)$. Inversely, for a slow response time laser $\left(\tau_{R}>>T_{\text {int }}\right)$, the stationary LOFI SNR is frequency dependent and is higher than the LOFI shot-noise limit at the laser relaxation frequency and smaller than the LOFI shot-noise limit far away from the laser relaxation frequency. Secondly, we have shown that the transient LOFI signal can be neglected compared to the stationary LOFI signal either by using a laser with a fast response time $\left(\tau_{R}<T_{\text {int }}\right.$ ) or by working with a slow response time laser $\left(\tau_{R}>T_{\text {int }}\right)$, if the frequency shift is far away from the relaxation frequency. Therefore to obtain a shot noise limited detection without any perturbation from the laser transient dynamics we need to work under the condition: $\tau_{R}<<T_{\text {int }}$.

Through this whole study, we have numerically confirmed that for a fixed integration time $\left(T_{\text {int }}\right)$, the best LOFI images (images with the best SNR) are always obtained when using the laser with the lowest LOFI gain, (i.e. the shortest laser time response $\tau_{R}$ ) and that the detection is shot noise limited if the following condition: $T_{\mathrm{int}}>\tau_{R}$ is satisfied.

Finally for fast imaging, we need a time value of $T_{\text {int }}$ as short as possible whereas to be shot noise limited we need to use a laser with a fast response time $\left(\tau_{R}<<T_{\text {int }}\right)$. For LOFI imaging the best laser is therefore a class-B laser with the shortest possible value of $\tau_{R}$, allowing the use of relatively short integration time $T_{\mathrm{int}}$. Therefore, for high speed imaging combined with a shotnoise limited detection, a laser diode with a very short response time (in the nanosecond range) and a very high value of the relaxation frequency (in the gigahertz range) seems to be an 
interesting candidate compared to microchip laser with a response time of several tens of microsecond and a relaxation frequency in the megahertz range. 


\section{REFERENCES}

1. T. Yoshizawa, editor, Handbook of optical metrology: Principles and Applications (CRC Press, 2009).

2. K. Otsuka, "Effects of external perturbations on $\mathrm{LiNdP}_{4} 0_{12}$ Lasers," IEEE J. Quantum Electron., QE-15, 655-663 (1979).

3. K. Otsuka, "Self-Mixing Thin-Slice Solids-State Laser Metrology," Sensors 11, 2195-2245 (2011).

4. K. Otsuka, "Highly sensitive measurement of Doppler-shift with a microchip solid-state laser,” Jpn. J. Appl. Phys. 31, L1546-L1548 (1992).

5. S. Okamoto, H. Takeda, and F. Kannari, "Ultrahighly sensitive laser-Doppler velocity meter with a diode-pumped Nd:YVO4 microchip laser,” Rev. Sci. Instrum. 66, 3116-3120 (1995).

6. R. Kawai, Y. Asakawa, K. Otsuka, "Ultrahigh-Sensitivity Self-Mixing Laser Doppler Velocimetry with Laser-Diode-Pumped Microchip LiNdP ${ }_{4} \mathrm{O}_{12}$ Lasers," IEEE Photonics Technology Lett. 11, 706-708 (1999).

7. S. Suddo, T. Ohtomo, Y. Takahascvhi, T. Oishi, K. Otsuka, "Determination of velocity of self-mobile phytoplankton using a self thin-slice solid-state laser,” Appl. Opt. 48, 4049-4055 (2009).

8. K. Otsuka, K. Abe, J.Y. Ko, and T.S. Lim, "Real-time nanometer vibration measurement with self-mixing microchip solid-state laser,” Opt. Lett. 27, 1339-1341 (2002).

9. V. Muzet, E.Lacot, O. Hugon, Y. Gaillard, "Experimental comparison of shearography and laser optical feedback imaging for crack detection in concrete structures," Proc. SPIE 5856, 793-799 (2005). 
10. E. Lacot, and O. Hugon, "Phase-sensitive laser detection by frequency-shifted optical feedback,” Phys. Rev. A 70, 053824 (2004).

11. H. Gilles, S. Girard, M. Laroche, and A. Belarouci, "Near-field amplitude and phase measurements using heterodyne optical feedback on solid-state lasers," Opt. Lett. 33, 1-3 (2008).

12. S. Blaize, B. Bérenguier, I. Stéfanon, A. Bruyant, G. Lerondel, P. Royer, O. Hugon, O. Jacquin, and E. Lacot, “ Phase sensitive optical near-field mapping using frequency-shifted laser optical feedback interferometry,” Opt. Express 16, 11718-11726 (2008).

13. E. Lacot, R. Day, and F. Stoeckel, "Laser optical feedback tomography," Opt. Lett. 24, 744746 (1999).

14. A. Witomski, E. Lacot, O. Hugon, and O. Jacquin, "Synthetic aperture laser optical feedback imaging using galvanometric scanning,” Opt. Lett. 31, 3031-3033 (2006).

15. O. Hugon, I.A. Paun, C. Ricard, B. van der Sanden, E. Lacot, O. Jacquin, A. Witomski, "Cell imaging by coherent backscattering microscopy using frequency shifted optical feedback in a microchip laser," Ultramicroscopy 108, 523-528 (2008).

16. O. Hugon, F. Joud, E. Lacot, O. Jacquin, H. Guillet de Chatellus, Coherent microscopy by laser optical feedback imaging (LOFI) technique, Ultramicroscopy (2011) doi: 10.1016/j.ultramic.2011.08.004.

17. E. Lacot, R. Day, and F. Stoeckel, "Coherent laser detection by frequency-shifted optical feedback,” Phys. Rev. A 64, 043815 (2001).

18. E. Lacot, O. Jacquin, G. Roussely, O. Hugon, H. Guillet de Chatellus, "Comparative study of autodyne and heterodyne laser interferometry for imaging," J. Opt. Soc. Am. A 27, 2450$2458(2010)$. 
19. O. Jacquin, E. Lacot, W. Glastre, O. Hugon, H. Guillet de Chatellus, "Expérimetal comparison of autodyne and heterodyne laser interferometry using an Nd:YVO4 microchip laser,” J. Opt. Soc. Am. A 28, 1741-1746 (2011).

20. J.J. Zaykowski and A. Mooradian, "Single -frequency microchip Nd lasers," Opt. Lett. 1, 2426 (1989).

21. M.I. Kolobov, L. Davidovich, E. Giacobino, and C. Fabre, "Role of pumping statistics and dynamics of atomic polarization in quantum fluctuations of laser sources," Phys. Rev. A 47, 1431-1446 (1993).

22. A. Bramati, J.P. Hermier, V. Jost, E. Giacobino, L. Fulbert, E. Molva, and J.J. Aubert, “Effects of pump fluctuations on intensity noise of Nd:YVO4 microchip lasers,” Eur. Phys. J. D. 6, 513-521 (1999).

23. Y.I. Khanin, Principles of laser dynamics, (Elsevier, 1995).

24. The saturation effect observed numerically can't be obtained analytically, because Eq. (2) was obtained after a linearization of the set of Eqs. (1), i.e. far away from the saturation conditions.

25. To observe the SNR amplification predicts by Eq. (21), one could imagine decreasing the LOFI gain by decreasing the laser time response, but in this case, the amplification effect which is proportional to laser time response then falls to very small value hides by the laser quantum fluctuations. 